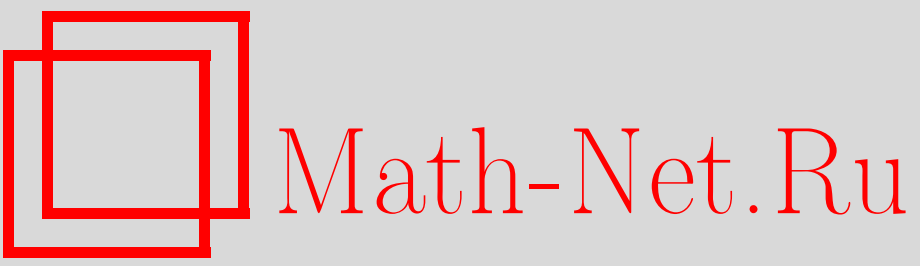

С. К. Немировский, Термодинамическое равновесие в системе хаотических квантованных вихрей в слабо неидеальном бозе-газе, ТМФ, 2004, том 141, номер 1, 141-151

DOI: https://doi.org/10.4213/tmf115

Использование Общероссийского математического портала Math-Net.Ru подразумевает, что вы прочитали и согласны с пользовательским соглашением

http: //www . mathnet.ru/rus/agreement

Параметры загрузки:

IP : 34.227 .88 .159

26 апреля 2023 г., 11:28:28 
ТЕОРЕТИЧЕСКАЯ

И МАТЕМАТИЧЕСКАЯ

ФИЗИКА

Том 141, № 1

октябрь, 2004

(C) 2004 г.

С.К. Немировский*

\section{ТЕРМОДИНАМИЧЕСКОЕ РАВНОВЕСИЕ В СИСТЕМЕ ХАОТИЧЕСКИХ КВАНТОВАННЫХ ВИХРЕЙ В СЛАБО НЕИДЕАЛЬНОМ БОЗЕ-ГАЗЕ}

На примере слабо неидеального бозе-газа обсуждается механизм установления термодинамического равновесия для хаотического набора квантовых вихревых нитей. Предполагается, что динамика бозе-конденсата описывается уравнением Гросса-Питаевского с добавочным шумом, удовлетворяющим флуктуационно-диссипативной теореме. При рассмотрении вихревой нити как линии пересечения поверхностей, на которых реальная и мнимая части параметра порядка $\psi(\mathbf{x}, t)$ обращаются в нуль, получается уравнение ланжевеновского типа для элементов вихревой нити с соответственно преобразованной случайной силой. При этом уравнение Фоккера-Планка для плотности вероятности имеет решение в форме распределения Гиббса с температурой бозе-конденсата. Другими словами, когда бозе-конденсат находится в тепловом равновесии и нет никаких других случайных воздействий, система вихрей также находится в тепловом равновесии.

Ключевые слова: квантовые вихревые нити, бозе-газ, термодинамическое равновесие, сверхтекучая турбулентность.

\section{1. ВВЕДЕНИЕ}

Квантованные вихри играют фундаментальную роль при описании квантовых жидкостей, сверхпроводников и других систем. Фазовые преврашения, кинетические свойства, а также множество других физических явлений связаны с появлением в системах квантованных вихрей и с их динамикой. По этой причине квантованные вихри являются объектом интенсивного изучения в течение многих лет. Подробный обзор и библиографию можно найти в монографии [1]. Из-за сложной динамики вихревые нити могут развиваться в запутанные хаотические структуры, называемые вихревыми клубками (vortex tangle). Известно, что система хаотических вихревых нитей может находиться как в состоянии термодинамического равновесия, что является объектом многочисленных исследований, особенно в космологическом контексте (см., например, [2]), так и в сильно неравновесном турбулентном состоянии (см., например, [1] и обзор [3]). Естественно возникает вопрос: какого рода состояние реализуется в каждом конкретном

* Институт теплофизики СО РАН, Новосибирск, Россия. E-mail: nemir@itp.nsc.ru 
эксперименте, и какого рода теорию следует использовать при описании тех или иных явлений? Ответ на такой вопрос может дать исследование, посвященное изучению конкретных механизмов, ответственных за установление термодинамического равновесия, и механизмов, описываюших переход в турбулентное состояние.

Предположение о тепловом равновесии может быть основано на фундаментальных физических принщипах, если рассматривать квантованные вихри как подсистему, погруженную в термостат, с которым вихри обмениваются энергией. Роль термостата в данном случае играет соответствуюшее физическое поле, образуюшее сверхтекучую компоненту. В случае бозе-конденсата, который мы рассматриваем в данной статье, роль такого поля играет параметр порядка $\psi(\mathbf{x}, t)$. Возбуждения параметра порядка (фононы, а в случае реального гелия также и ротоны) взаимодействуют с ансамблем вихревых петель, приводя последний к статистическому распределению, котороев соответствии с обшим принципом максимальности энтропии должно быть распределением Гиббса. Приведенное рассуждение, однако, не раскрьвает никаких деталей динамики системы. Иными словами, оно не отвечает на вопрос, каким образом система вихрей приводится к термодинамическому равновесию. Между тем, понимая, какие конкретно механизмы ответственны за установление термодинамического равновесия, мы могли бы дать ответ и на другой интересующий нас вопрос: каким образом система вихрей переходит в сильно неравновесное (турбулентное) состояние?

В предлагаемой работе мы сосредоточимся на первом вопросе, касающемся термодинамического равновесия. С целью проследить динамические детали установления равновесия мы в рамках ланжевеновского подхода изучим, каким образом набор хаотических вихревых петель в равновесном бозе-конденсате приводится в тепловое равновесие.

Поставленная проблема решается в два этапа. На первом этапе (в разделе 2) мы, исходя из ланжевеновского описания стохастической динамики для параметра порядка $\psi(\mathbf{x}, t)$ слабо неидеального бозе-газа, выводим уравнения движения для вихревых линий, рассматривая последние как линии обрашения в нуль величины $\psi(\mathbf{x}, t)$, т.е. топологические дефекты. Полученное уравнение для динамики элементов вихревой нити $\mathbf{s}(\xi, t)$ является также уравнением ланжевеновского типа с соответственно преобразованной случайной силой и диссипативным членом. На основании этого уравнения мы выводим (в разделе 3) уравнение Фоккера-Планка для функционала вероятности $\mathcal{P}(\{\mathbf{s}(\xi)\}, t)$, описьваюшего вероятность того, что система вихревых петель составляет некоторую конфигурацию $\mathcal{P}(\{\mathbf{s}(\xi)\}, t)$. Далее мы показываем, что уравнение Фоккера-Планка имеет решение в виде распределения Гиббса $\mathcal{P}(\{\mathbf{s}(\xi)\}, t) \propto \exp (-H\{\mathbf{s}\} / T)$, где $H\{\mathbf{s}\}$ - функционал энергии данной конфигурации вихревых линий.

\section{2. УРАВНЕНИЕ ЛАНЖЕВЕНА ДЛЯ ПАРАМЕТРА ПОРЯДКА}

В этом разделе мы получаем уравнение движения квантованных вихрей в бозе-газе, динамика которого описывается уравнением Гросса-Питаевского [4], [5] с добавленной случайной (ланжевеновской) силой. В системе, где нормальная скорость отсутствует, 
это уравнение выглядит следующим образом:

$$
\frac{\partial \psi}{\partial t}=-(\Lambda+i) \frac{\delta H(\psi)}{\delta \psi^{*}}+f(\mathbf{x}, t)
$$

где $H\{\psi\}$ - функционал свободной энергии Гинзбурга-Ландау

$$
H\{\psi\}=\int d^{3} x\left[\frac{\hbar^{2}}{2 m^{2}}|\nabla \psi|^{2}-\frac{\mu}{m}|\psi|^{2}+\frac{V_{0}}{2 m}|\psi|^{4}\right] .
$$

В выписанных соотношениях приняты следуюшие обозначения: $\Lambda$ - безразмерньй кинетический коэффициент, $\mu$ - химический потенциал, $V_{0}$ - потенциал, описывающий взаимодействие частиц, $m$ - масса частицы. Параметр порядка $\psi=\sqrt{\rho_{s}} e^{i \phi}$, где $\rho_{s}-$ плотность бозе-конденсата, а фаза $\phi$ определена таким образом, что скорость потока $\mathbf{v}=$ $(\hbar / m) \nabla \psi$. Масштаб времени в уравнении $(1)$ изменен по правилу $t \rightarrow t(\hbar / m)$. Тепловой шум $f(\mathbf{x}, t)$ подчиняется флуктуационно-диссипативной теореме

$$
\left\langle f\left(\mathbf{x}_{1}, t_{1}\right) f^{*}\left(\mathbf{x}_{2}, t_{2}\right)\right\rangle=2 k_{\mathrm{B}} T \Lambda \delta\left(\mathbf{x}_{1}-\mathbf{x}_{2}\right) \delta\left(t_{1}-t_{2}\right) .
$$

Сформулированная стохастическая проблема имеет решение, описываюшее тепловое равновесие, где вероятность некоторой конфигурации поля $\{\psi(\mathbf{x})\}$ удовлетворяет закону Гиббса. Это следует из решения соответствуюшего уравнения Фоккера-Планка [6].

Перейдем к проблеме получения динамики вихрей, появляюшихся в бозе-конденсате, эволюция которого описывается уравнением Гросса-Питаевского (1). Эта проблема относится к числу обших проблем получения уравнения движения топологических дефектов (доме́нных стенок, дислокаций, космических струн и т.д.) на основе нелинейной динамики полей, порождающих эти дефекты. В контексте движения вихревых нитей в квантовых жидкостях такая задача исследовалась ранее, начиная с работы [7] (см. также монографию [8]). В этих работах, однако, исследовались детерминистские уравнения. В предлагаемой статье изложим новый метод, удобный для преобразования случайного шума $f(\mathbf{x}, t)$, действуюшего на бозе-газ, в случайную скорость для элементов вихревой нити.

Вихри в квантовых жидкостях представляют собой очень тонкие вихревые трубки, и их динамика аналогична динамике струн. Таким образом, мы должны исключить радиальные степени свободы, что может быть достигнуто с помощью следующей процедуры. Эволюция вихревых нитей (как линий пересечения нулевых поверхностей параметра порядка) в квантовых жидкостях определяется динамикой функции $\psi(\mathbf{x}, t)$, т.е. уравнением Гросса-Питаевского (1). С другой стороны, если не рассматривать другие степени свободы (фононы), можно утверждать, что все движение бозе-конденсата определено динамикой вихревых нитей. Таким образом, имеется взаимное соответствие, и параметр порядка $\psi(\mathbf{x}, t)$ может рассматриваться как некоторый функционал от полной вихревой конфигурации, т.е. $\psi(\mathbf{x}, t)=\psi(\mathbf{x},\{\mathbf{s}(\xi, t)\})$. Так, например, временна́я зависимость поля $\psi(\mathbf{x}, t)$ (в некоторой точке трехмерного пространства $\mathbf{x}$ ) связана с движением 
всей вихревой конфигурации $\mathbf{s}(\xi, t)$ и описьвается в соответствии со следующим цепным правилом:

$$
\frac{\partial \psi(\mathbf{x}, t)}{\partial t}=\int_{\Gamma} \frac{\delta \psi(\mathbf{x}, t)}{\delta \mathbf{s}\left(\xi^{\prime}, t\right)} \frac{\partial \mathbf{s}\left(\xi^{\prime}, t\right)}{\partial t} d \xi^{\prime} .
$$

Здесь и в последуюшем мы обозначаем индексом Г контурные интегралы вдоль отдельных линий и суммирование по всем вихревым петлям.

Перейдем к выводу уравнения движения вихревых нитей. Перепишем уравнение (1) в следующей форме:

$$
\frac{\Lambda-i}{\Lambda^{2}+1} \frac{\partial \psi}{\partial t}=-\frac{\delta H(\psi)}{\delta \psi^{*}}+\frac{\Lambda-i}{\Lambda^{2}+1} f(\mathbf{x}, t) .
$$

Умножим уравнение (5) на $\delta \psi^{*} / \delta \mathbf{s}\left(\xi_{0}, t\right)$, где $\xi_{0}$ - некоторая выбранная точка на кривой. Складывая результат с комплексно-сопряженным и интегрируя по всему пространству, получим

$$
\begin{aligned}
\int d^{3} \mathbf{x} & \left(\frac{\Lambda-i}{\Lambda^{2}+1} \frac{\partial \psi}{\partial t} \frac{\delta \psi^{*}}{\delta \mathbf{s}\left(\xi_{0}, t\right)}+\frac{\Lambda+i}{\Lambda^{2}+1} \frac{\partial \psi^{*}}{\partial t} \frac{\delta \psi}{\delta \mathbf{s}\left(\xi_{0}, t\right)}\right)= \\
= & -\int d^{3} \mathbf{x}\left(\frac{\delta H\left(\psi, \psi^{*}\right)}{\delta \psi^{*}} \frac{\delta \psi^{*}}{\delta \mathbf{s}\left(\xi_{0}, t\right)}+\frac{\delta H\left(\psi, \psi^{*}\right)}{\delta \psi^{*}} \frac{\delta \psi}{\delta \mathbf{s}\left(\xi_{0}, t\right)}\right)+ \\
& +\int d^{3} \mathbf{x}\left(\frac{\Lambda-i}{\Lambda^{2}+1} f(\mathbf{x}, t) \frac{\delta \psi^{*}}{\delta \mathbf{s}\left(\xi_{0}, t\right)}+\frac{\Lambda+i}{\Lambda^{2}+1} f^{*}(\mathbf{x}, t) \frac{\delta \psi}{\delta \mathbf{s}\left(\xi_{0}, t\right)}\right)
\end{aligned}
$$

Первый интеграл в правой части этого соотношения следует из цепного правила для функциональной производной $\delta H(\mathbf{s}) / \delta \mathbf{s}\left(\xi_{0}, t\right)$, где $H(\mathbf{s})$ - энергия бозе-конденсата, выраженная через полную вихревую конфигурацию. Последовательно рассматривая вихри очень тонкими трубками (что оправданно, когда радиус кривизны $R$ намного превышает размер ядра $\left.r_{0}\right)$ и пренебрегая энергией, связанной с ядром, запишем кинетическую энергию сверхтекучего движения, созданного вихрями (см., например, [9]):

$$
H(\mathbf{s})=\frac{\rho_{s} \kappa^{2}}{8 \pi} \int_{\Gamma} \int_{\Gamma^{\prime}} \frac{\mathbf{s}^{\prime}(\xi) \mathbf{s}^{\prime}\left(\xi^{\prime}\right)}{\left|\mathbf{s}(\xi)-\mathbf{s}\left(\xi^{\prime}\right)\right|} d \xi d \xi^{\prime} .
$$

Здесь $\mathbf{s}^{\prime}(\xi)$ - тангенциальный вектор вдоль вихревой линии, $\kappa$ - квант циркуляции, равньй $2 \pi \hbar / m$. Вычисление функциональной производной дает

$$
\frac{\delta H(\mathbf{s})}{\delta \mathbf{s}\left(\xi_{0}, t\right)}=\rho_{s} \kappa \mathbf{s}^{\prime}\left(\xi_{0}\right) \times \mathbf{B}\left(\xi_{0}\right) .
$$

Величина $\mathbf{B}\left(\xi_{0}\right)$ - скорость элемента вихревой нити в точке $\boldsymbol{\xi}_{0}$, выраженная с помощью известного закона Био-Савара:

$$
\mathbf{B}\left(\xi_{0}\right)=-\frac{\kappa}{4 \pi} \int_{\Gamma^{\prime}} \frac{\left(\mathbf{s}\left(\xi_{0}\right)-\mathbf{s}\left(\xi^{\prime}\right)\right) \times \mathbf{s}^{\prime}(\xi)}{\left|\mathbf{s}\left(\xi_{0}\right)-\mathbf{s}\left(\xi^{\prime}\right)\right|^{3}} d \xi^{\prime}
$$

Таким образом, мы записали интересуюший нас интеграл в соотношении (6) только в терминах переменной $\mathbf{s}(\xi, t)$, описывающей вихревую конфигурацию. 
Рассмотрим интеграл в левой части уравнения (6). Подставив соотношение (4) для временно́й производной $\partial \psi / \partial t$, а также соответствуюшее комплексно-сопряженное выражение, в подынтегральном выражении получим

$$
\frac{\Lambda-i}{\Lambda^{2}+1} \int d^{3} \mathbf{x} \int_{\Gamma^{\prime}}\left(\frac{\delta \psi(\mathbf{x} \mid \mathbf{s}(\xi, t))}{\delta \mathbf{s}\left(\xi^{\prime}, t\right)} \frac{\partial \mathbf{s}\left(\xi^{\prime}, t\right)}{\partial t}\right) \frac{\delta \psi^{*}(\mathbf{x} \mid \mathbf{s}(\xi, t))}{\delta \mathbf{s}\left(\xi_{0}, t\right)} d \xi^{\prime}+\text { к. c. }
$$

Интеграл (10) зависит от полной вихревой конфигурации. Однако этот интеграл (и другие подобные, включающие квадратичные выражения от градиентов параметра порядка) может быть вычислен приближенно в общей форме при оговоренном условии, что радиус кривизны нити сушественно превышает размер ядра (см., например, [8]). Соответствуюшие расчеты содержатся в приложении. Интересующий нас интеграл представляет сумму выражений (П.4) и (П.9), т.е. левая часть уравнения (6) принимает вид

$$
\frac{2 \pi \rho_{s}}{\Lambda^{2}+1} \dot{\mathbf{s}}\left(\xi_{0}\right) \times \mathbf{s}^{\prime}\left(\xi_{0}\right)+\frac{2 \pi \rho_{s} \sigma \Lambda}{\Lambda^{2}+1} \dot{\mathbf{s}}\left(\xi_{0}\right)
$$

Таким образом, мы опять записали интересуюший нас интеграл в соотношении (6) в терминах переменной $\mathbf{s}(\xi, t)$, описываюшей вихревую конфигурацию.

Обсудим теперь второе слагаемое в правой части уравнения (6), включающее случайную силу $f(\mathbf{x}, t)$. Последовательно считая, что все движение бозе-конденсата связано с движением вихрей (напомним, что мы не рассматриваем акустические эффекты), можно представить случайную силу, фигурируюшую в уравнении (6), как некоторую "вторичную" величину, возникшую от случайных смешений (всех) элементов (всех) нитей, т.е. малые случайные вариации параметра порядка $\delta \psi$ связаны с малыми случайными вариациями смещений элементов нити $\delta \mathbf{s}$ с помощью цепного правила

$$
\delta \psi(\mathbf{x}, t)=\int_{\Gamma} \frac{\delta \psi(\mathbf{x}, t)}{\delta \mathbf{s}\left(\xi^{\prime}, t\right)} \delta \mathbf{s}\left(\xi^{\prime}, t\right) d \xi^{\prime}
$$

Малые вариации $\delta \psi$ и $\delta \mathbf{s}$ за некоторый интервал времени $\delta t$ связаны со случайными силами очевидным образом:

$$
\begin{aligned}
\delta \mathbf{s}(\xi, t) & =\mathbf{f}(\xi, t) \delta t, \\
\delta \psi(\mathbf{x}, t) & =f(\mathbf{x}, t) \delta t .
\end{aligned}
$$

Следует различать случайную силу $f(\mathbf{x}, t)$, действуюшую на параметр порядка, и случайную скорость $\mathbf{f}(\xi, t)$.

Подставляя (13) и (14) в (12) и сокрашая на $\delta t$, получим связь между случайной силой $f(\mathbf{x}, t)$ и случайной скоростью $\mathbf{f}(\xi, t)$ :

$$
f(\mathbf{x}, t)=\int_{\Gamma} \frac{\delta \psi(\mathbf{x}, t)}{\delta \mathbf{s}\left(\xi^{\prime}, t\right)} \mathbf{f}\left(\xi^{\prime}, t\right) d \xi^{\prime}
$$


Подставив это выражение (а также соответствуюшее комплексно-сопряженное) во второй интеграл в правой части уравнения (6), получим

$$
\frac{\Lambda-i}{\Lambda^{2}+1} \int d^{3} \mathbf{x} \int_{\Gamma^{\prime}}\left(\frac{\delta \psi(\mathbf{x} \mid s(\xi, t))}{\delta \mathbf{s}\left(\xi^{\prime}, t\right)} \mathbf{f}\left(\xi^{\prime}, t\right)\right) \frac{\delta \psi^{*}(\mathbf{x} \mid \mathbf{s}(\xi, t))}{\delta \mathbf{s}\left(\xi_{0}, t\right)} d \xi^{\prime}+\text { к. с. }
$$

Но это выражение совпадает с выражением (10) после очевидной замены $\dot{\mathbf{s}}\left(\xi_{0}\right) \rightarrow \mathbf{f}\left(\xi_{0}, t\right)$. Применяя технику, описанную в приложении, мы можем записать интеграл (16) в виде

$$
\frac{2 \pi}{\Lambda^{2}+1} \mathbf{f}\left(\xi_{0}, t\right) \times \mathbf{s}^{\prime}\left(\xi_{0}\right)+\frac{2 \pi \Lambda}{\Lambda^{2}+1} \mathbf{f}\left(\xi_{0}, t\right) .
$$

Таким образом, уравнение (6) можно записать как

$$
\begin{aligned}
& \frac{1}{\Lambda^{2}+1} \dot{\mathbf{s}}\left(\xi_{0}\right) \times \mathbf{s}^{\prime}\left(\xi_{0}\right)+\frac{\Lambda \sigma}{\Lambda^{2}+1} \dot{\mathbf{s}}\left(\xi_{0}\right)= \\
& \quad=\mathbf{s}^{\prime}\left(\xi_{0}\right) \times \mathbf{B}\left(\xi_{0}\right)+\frac{1}{\Lambda^{2}+1} \mathbf{f}\left(\xi_{0}, t\right) \times \mathbf{s}^{\prime}\left(\xi_{0}\right)+\frac{\Lambda \sigma}{\Lambda^{2}+1} \mathbf{f}\left(\xi_{0}, t\right)
\end{aligned}
$$

Это векторное уравнение может быть разрешено относительно величины $\dot{\mathbf{s}}\left(\xi_{0}\right)$ с точностью до составляющей $\dot{\mathbf{s}}_{\|}\left(\xi_{0}\right)$ вдоль кривой, которая не имеет физического значения и может быть удалена подходящей параметризацией переменной $\xi$. Результат имеет следующий вид:

$$
\dot{\mathbf{s}}\left(\xi_{0}\right)=\frac{1+\Lambda^{2}}{1+\Lambda^{2} \sigma^{2}} \mathbf{B}\left(\xi_{0}\right)+\frac{\left(1+\Lambda^{2}\right) \Lambda \sigma}{1+\Lambda^{2} \sigma^{2}} \mathbf{s}^{\prime}\left(\xi_{0}\right) \times \mathbf{B}\left(\xi_{0}\right)+\mathbf{f}\left(\xi_{0}, t\right) .
$$

Уравнение (19) описьвает движение элементов вихревой линии в терминах самой вихревой линии. Замечателен тот факт (не очевидный заранее), что шум $\mathbf{f}\left(\xi_{0}, t\right)$, действующий на линии, также является аддитивным.

Установим теперь статистические свойства шума $\mathbf{f}\left(\xi_{0}, t\right)$ и его интенсивность. Выражение (17) является результатом преобразования второго слагаемого в правой части уравнения (6), т.е.

$$
\begin{aligned}
& \frac{\hbar}{m} \frac{2 \pi \rho_{s}}{\Lambda^{2}+1} \mathbf{f}\left(\xi_{1}, t_{1}\right) \times \mathbf{s}^{\prime}\left(\xi_{1}\right)+\frac{\hbar}{m} \frac{2 \pi \rho_{s} \Lambda}{\Lambda^{2}+1} \mathbf{f}\left(\xi_{1}, t_{1}\right)= \\
& \quad=\int d^{3} \mathbf{x}_{1}\left(\frac{\Lambda-i}{\Lambda^{2}+1} f\left(\mathbf{x}_{1}, t_{1}\right) \frac{\delta \psi^{*}}{\delta \mathbf{s}\left(\xi_{1}, t\right)}+\frac{\Lambda+i}{\Lambda^{2}+1} f^{*}\left(\mathbf{x}_{1}, t_{1}\right) \frac{\delta \psi}{\delta \mathbf{s}\left(\xi_{1}, t\right)}\right)
\end{aligned}
$$

Умножив скалярно обе части уравнения (20) на соответствуюшие части такого же уравнения, но записанного для другого момента времени $t_{2}$ и другой точки $\xi_{2}$, получим

$$
\begin{aligned}
4 \pi^{2} & \frac{\hbar^{2}}{m^{2}} \frac{1+\Lambda^{2} \sigma^{2}}{\left(\Lambda^{2}+1\right)^{2}}\left(\mathbf{f}_{\eta_{1}}\left(\xi_{1}, t_{1}\right) \mathbf{f}_{\eta_{1}}\left(\xi_{2}, t_{2}\right)+\mathbf{f}_{\eta_{2}}\left(\xi_{1}, t_{1}\right) \mathbf{f}_{\eta_{2}}\left(\xi_{2}, t_{2}\right)\right)= \\
= & \frac{2}{\Lambda^{2}+1} \iint d^{3} \mathbf{x}_{1} d^{3} \mathbf{x}_{2} \frac{\delta \psi\left(\mathbf{x}_{1}, t_{1}\right)}{\delta \mathbf{s}\left(\xi_{1}, t_{1}\right)} \frac{\delta \psi^{*}\left(\mathbf{x}_{2}, t_{2}\right)}{\delta \mathbf{s}\left(\xi_{2}, t_{2}\right)} f\left(\mathbf{x}_{1}, t_{1}\right) f^{*}\left(\mathbf{x}_{2}, t_{2}\right) .
\end{aligned}
$$


Здесь $\mathbf{f}_{\eta_{1}}$ и $\mathbf{f}_{\eta_{2}}$ - компоненты случайных скоростей во взаимно перпендикулярных направлениях $\eta_{1}, \eta_{2}$, лежащих в плоскости, нормальной к линии. Мы опустили остальные слагаемые в правой части выражения (21), предвидя, что они исчезнут после усреднения. Усредняя (21) и используя флуктуационно-диссипативную теорему (3), а также вычисляя трехмерные интегралы по правилам, описанным в приложении, получаем

$$
\left\langle\mathbf{f}_{\eta_{1}}\left(\xi_{1}, t_{1}\right) \mathbf{f}_{\eta_{2}}\left(\xi_{2}, t_{2}\right)\right\rangle=\frac{k_{\mathrm{B}} T}{\rho_{s} \pi(\hbar / m)} \frac{\left(\Lambda^{2}+1\right) \Lambda \sigma}{1+\Lambda^{2} \sigma^{2}} \delta\left(\xi_{1}-\xi_{2}\right) \delta\left(t_{1}-t_{2}\right) \delta_{\eta_{1} \eta_{2}} .
$$

Таким образом, исходя из ланжевеновской динамики бозе-конденсата (соотношения $(1),(3))$, мы получили уравнения (19) и (22), описывающие ланжевеновскую динамику вихревой нити непосредственно в терминах самой нити. Выписанные соотношения (19) и (22) завершают формулировку проблемы стохастической динамики квантованных вихрей, взаимодействующих с термостатом. Роль термостата играют тепловые возбуждения параметра порядка. В следующем разделе мы покажем, что эта проблема имеет стационарное решение в виде равновесного распределения Гиббса $\exp \left(-H\{\mathbf{s}\} / k_{\mathrm{B}} T\right)$, где $H\{\mathbf{s}\}-$ энергия вихревой системы (см. уравнение $(7))$.

\section{3. УРАВНЕНИЕ ФОККЕРА-ПЛАНКА}

Получим сначала уравнение Фоккера-Планка, соответствующее ланжевеновской динамике, описывающейся соотношениями (19) и (22). Введем следующий функционал распределения вероятности:

$$
\mathcal{P}(\{\mathbf{s}(\xi)\}, t)=\left\langle\mathcal{P}^{M}\right\rangle=\langle\delta(\mathbf{s}(\xi)-\mathbf{s}(\xi, t))\rangle
$$

Здесь $\delta$ является дельта-функционалом в пространстве конфигураций вихревых петель. Усреднение выполнено по ансамблю случайной силы. Эволюция величины $\mathcal{P}(\{\mathbf{s}(\xi)\}, t)$ может быть представлена в виде

$$
\frac{\partial \mathcal{P}}{\partial t}=\left\langle-\int d \xi\left\{\frac{\delta \mathcal{P}^{M}}{\delta \mathbf{s}(\xi)} \dot{\mathbf{s}}_{\mathrm{det}}\right\}\right\rangle-\int d \xi\left\{\frac{\delta}{\delta \mathbf{s}(\xi)}\left\langle\mathbf{f}(\xi, t) \mathcal{P}^{M}\right\rangle\right\},
$$

где $\dot{\mathbf{s}}_{\mathrm{det}}-$ детерминированная часть скорости, определенная уравнением (19). Опуская стандартные детали (см., например, [10]), запишем следующее из выражения (24) уравнение Фоккера-Планка:

$$
\begin{aligned}
\frac{\partial \mathcal{P}}{\partial t} & +\int d \xi \frac{\delta}{\delta \mathbf{s}(\xi)}\left\{\left[\frac{1+\Lambda^{2}}{1+\Lambda^{2} \sigma^{2}} \mathbf{B}(\xi)+\frac{\left(1+\Lambda^{2}\right) \Lambda \sigma}{1+\Lambda^{2} \sigma^{2}} \mathbf{s}^{\prime}(\xi) \times \mathbf{B}(\xi)\right] \mathcal{P}\right\}+ \\
& +\int d \xi \int d \xi^{\prime}\left\langle\mathbf{f}(\xi) \mathbf{f}\left(\xi^{\prime}\right)\right\rangle \frac{\delta}{\delta \mathbf{s}\left(\xi^{\prime}\right)} \mathcal{P}=0 .
\end{aligned}
$$

В формуле (25) $\left\langle\mathbf{f}(\xi) \mathbf{f}\left(\xi^{\prime}\right)\right\rangle$ - корреляционная функция случайной силы, действующей на линию (см. уравнение (22)). Наша цель теперь состоит в том, чтобы доказать, что уравнение (25) имеет стационарное решение в виде $\mathcal{P}(\{\mathbf{s}(\xi)\})=\mathcal{N} \exp \left(-H\{\mathbf{s}\} / k_{\mathrm{B}} T\right)$, 
где $\mathcal{N}$ - нормировочный множитель, $H\{\mathbf{s}\}$ - энергия вихревой системы (см. уравнение (7)).

Рассмотрим первое слагаемое в подынтегральном выражении уравнения (25). Используя параметризацию, в которой скорость $\dot{\mathbf{s}}\left(\xi_{0}\right)$ нормальна вихревой нити, перепишем выражение (8) в виде

$$
\mathbf{B}(\xi)=\frac{1}{\rho_{s} \kappa} \mathbf{s}^{\prime}(\xi) \times \frac{\delta H(\mathbf{s})}{\delta \mathbf{s}\left(\xi_{0}, t\right)} .
$$

Тогда первое слагаемое в подынтегральном выражении уравнения (25) примет вид

$$
\mathcal{N} \frac{1}{\rho_{s} \kappa} \frac{1+\Lambda^{2}}{1+\Lambda^{2} \sigma^{2}} \int d \xi \frac{\delta}{\delta \mathbf{s}(\xi)}\left(\mathbf{s}^{\prime}(\xi) \times \frac{\delta H(\mathbf{s})}{\delta \mathbf{s}(\xi, t)} \exp \left(-\frac{H\{\mathbf{s}\}}{k_{\mathrm{B}} T}\right)\right) .
$$

Выполняя функциональное дифференцирование и используя тензорные обозначения, перепишем (27) в следуюшем виде (опуская коэффициент перед интегралом):

$$
\begin{aligned}
& \int d \xi \exp \left(-\frac{H\{\mathbf{s}\}}{k_{\mathrm{B}} T}\right) \epsilon^{\alpha \beta \gamma} \frac{\delta \mathbf{s}_{\beta}^{\prime}(\xi)}{\delta \mathbf{s}_{\alpha}(\xi)} \frac{\delta H(\mathbf{s})}{\delta \mathbf{s}_{\gamma}(\xi, t)}+ \\
& +\int d \xi \exp \left(-\frac{H\{\mathbf{s}\}}{k_{\mathrm{B}} T}\right) \epsilon^{\alpha \beta \gamma}\left(\mathbf{s}_{\beta}^{\prime}(\xi) \frac{\delta}{\delta \mathbf{s}_{\alpha}(\xi)} \frac{\delta H(\mathbf{s})}{\delta \mathbf{s}_{\gamma}(\xi, t)}\right)+ \\
& +\frac{1}{k_{\mathrm{B}} T} \int d \xi \exp \left(-\frac{H\{\mathbf{s}\}}{k_{\mathrm{B}} T}\right) \epsilon^{\alpha \beta \gamma} \mathbf{s}_{\beta}^{\prime}(\xi) \frac{\delta H(\mathbf{s})}{\delta \mathbf{s}_{\gamma}(\xi, t)} \frac{\delta H(\mathbf{s})}{\delta \mathbf{s}_{\alpha}(\xi, t)}
\end{aligned}
$$

Производная оператора $\delta \mathbf{s}_{\beta}^{\prime}(\xi) / \delta \mathbf{s}_{\alpha}(\xi) \propto \delta_{\beta \alpha}$, поэтому все члены тождественно исчезают из-за симметрии. Таким образом, обратимый (не связанный с диссипацией) член в исходном уравнении движения (19) не дает вклада в динамику функционала распределения вероятности. Понятно, что сказанное вьше относится только к случаю теплового равновесия, т.е. справедливо только для распределения Гиббса.

Последнее слагаемое в подынтегральном выражении (25) может быть преобразовано с помошью флуктуационно-диссипативной теоремы для линии (22) следующим образом:

$$
\begin{aligned}
& \int d \xi \int d \xi^{\prime}\left\langle\mathbf{f}(\xi) \mathbf{f}\left(\xi^{\prime}\right)\right\rangle \frac{\delta}{\delta \mathbf{s}\left(\xi^{\prime}\right)} \mathcal{P}= \\
& =\int d \xi \int d \xi^{\prime} \frac{k_{\mathrm{B}} T}{\pi} \frac{\left(\Lambda^{2}+1\right) \Lambda \sigma}{1+\Lambda^{2} \sigma^{2}} \times \\
& \quad \times \delta\left(\xi_{1}-\xi_{2}\right) \delta\left(t_{1}-t_{2}\right) \delta_{\eta_{1}, \eta_{2}} \frac{\delta}{\delta \mathbf{s}\left(\xi^{\prime}\right)} \exp \left(-\frac{H\{\mathbf{s}\}}{k_{\mathrm{B}} T}\right)
\end{aligned}
$$

Воспользовавшись снова соотношениями (8) и (26), легко убедиться, что получившееся выражение точно компенсирует второе слагаемое в подынтегральном выражении уравнения (25) (их сумма рана нулю). Таким образом, интегральный член в уравнении (25) исчезает. Это означает, что распределение Гиббса действительно является решением уравнения Фоккера-Планка, как это и должно быть на основании общих физических принципов. 


\section{4. ЗАКЛЮЧЕНИЕ}

Исследуя ланжевеновскую динамику хаотического набора квантовых вихревых нитей, возникающих в слабо неидеальном бозе-газе, мы проследили механизм установления термодинамического равновесия в вихревом клубке. Анализируя наше доказательство, можно видеть, что основная причина установления равновесия состоит в том, что аддитивная случайная сила $f(\mathbf{x}, t)$, действующая на параметр порядка, трансформируется в аддитивную случайную скорость $\mathbf{f}(\xi, t)$, соответствуюшую элементам вихревой нити. При этом закон трансформации случайной силы согласуется с законом трансформации диссипативного слагаемого таким образом, что в уравнении ФоккераПланка слагаемое, ответственное за накачку энергии, точно компенсирует диссипативньй поток распределения вероятности. Причем эта компенсация происходит в подынтегральном выражении. Иными словами, реализуется детальный баланс меж ду накачкой и диссипацией. Из этого следует, что при возникновении других источников случайного воздействия на систему (например, при развитии неустойчивости в текущем/противотекущем Не II) возможен переход в неравновесное (турбулентное) состояние. Соответствующее исследование, однако, выходит за рамки этой статьи.

\section{ПРИЛОЖЕНИЕ}

Главный вклад в интегралы типа (10) вносят точки, близкие к вихревой нити. Вклад от удаленных точек приводит к слабой логарифмической расходимости, и радиус кривизны (или межвихревое расстояние) используется обычно как верхний параметр обрезания. Практически это подразумевает, что можно заменить функционал $\psi(\mathbf{x} \mid \mathbf{s}(\xi, t))$ выражением $\psi_{v}\left(\mathbf{x}_{\perp}\right)=\psi_{v}\left(\mathbf{s}\left(\xi_{\mathrm{cl}}, t\right)-\mathbf{x}\right)$, где $\xi_{\mathrm{cl}}$ - точка на линии, ближайшая к точке $\mathbf{x}$ трехмерного пространства. Функция $\psi_{v}\left(\mathbf{x}_{\perp}\right)$ есть хорошо известное двумерное статическое решение, описываюшее двумерный вихрь (см., например, [8]). Интегрирование по $d^{3} \mathbf{x}$ может быть заменено на $d^{2} \mathbf{x}_{\perp} d \xi_{\mathrm{cl}}$. Тогда, например, функциональная производная $\delta \psi^{*} / \delta \mathbf{s}\left(\xi^{\prime}, t\right)$ вычисляется в соответствии с правилом

$$
\frac{\delta \psi(\mathbf{x} \mid \mathbf{s}(\xi, t))}{\delta \mathbf{s}\left(\xi^{\prime}, t\right)}=\nabla_{\perp} \psi_{v}\left(\mathbf{x}_{\perp}\right) \delta\left(\xi^{\prime}-\xi_{\mathrm{cl}}\right) .
$$

Аналогичное выражение может быть выписано для $\delta \psi^{*} / \delta \mathbf{s}\left(\xi_{0}, t\right)$. В результате интегрирование по $d \xi_{\mathrm{cl}}$ и $d \xi^{\prime}$ устраняет все дельта-функции, и выражение (10) принимает вид

$$
\frac{\Lambda-i}{\Lambda^{2}+1} \int d^{2} \mathbf{x}_{\perp}\left(\dot{\mathbf{s}}\left(\xi_{0}\right) \nabla_{\perp} \psi_{v}\left(\mathbf{x}_{\perp}\right)\right) \nabla_{\perp} \psi_{v}^{*}\left(\mathbf{x}_{\perp}\right)+\text { к. c. }
$$

где $\dot{\mathbf{s}}\left(\xi_{0}\right)$ - скорость линии в точке наблюдения $\xi_{0}$. Выражение (П.1) разлагается на сумму и разность одинаковых выражений. Разность может быть переписана с помощью известной формулы векторной алгебры:

$$
\frac{i}{\Lambda^{2}+1} \int d^{2} \mathbf{x}_{\perp} \dot{\mathbf{s}}\left(\xi_{0}\right) \times\left(\nabla_{\perp} \psi_{v}^{*}\left(\mathbf{x}_{\perp}\right) \times \nabla_{\perp} \psi_{v}\left(\mathbf{x}_{\perp}\right)\right) .
$$


Для выгисления (П.2) воспользуемся записью статического решения $\psi_{v}\left(\mathbf{x}_{\perp}\right)$ в локальных полярных координатах $\psi_{v}\left(\mathbf{x}_{\perp}\right)=A\left(\left|\mathbf{x}_{\perp}\right|\right) e^{i \varphi}$. Векторное произведение имеет вид

$$
\nabla_{\perp} \psi_{v}^{*}\left(\mathbf{x}_{\perp}\right) \times \nabla_{\perp} \psi_{v}\left(\mathbf{x}_{\perp}\right)=2 i \frac{A\left(\left|\mathbf{x}_{\perp}\right|\right) A^{\prime}\left(\left|\mathbf{x}_{\perp}\right|\right)}{\left|\mathbf{x}_{\perp}\right|} \mathbf{s}^{\prime}\left(\xi_{0}\right)
$$

и входящий в (П.2) интеграл вычисляется следующим образом:

$$
\begin{aligned}
& \int d^{2} \mathbf{x}_{\perp}\left(\nabla_{\perp} \psi_{v}^{*}\left(\mathbf{x}_{\perp}\right) \times \nabla_{\perp} \psi_{v}\left(\mathbf{x}_{\perp}\right)\right)= \\
& \quad=\mathbf{s}^{\prime}\left(\xi_{0}\right) \int 2 i \frac{A\left(\left|\mathbf{x}_{\perp}\right|\right) A^{\prime}\left(\left|\mathbf{x}_{\perp}\right|\right)}{\left|\mathbf{x}_{\perp}\right|} 2 \pi\left|\mathbf{x}_{\perp}\right| d\left|\mathbf{x}_{\perp}\right|=2 \pi i \rho_{s} \mathbf{s}^{\prime}\left(\xi_{0}\right)
\end{aligned}
$$

Таким образом, искомая часть $\partial \psi / \partial t$, представленная выражением (П.2), имеет вид

$$
\frac{2 \pi \rho_{s}}{\Lambda^{2}+1} \dot{\mathbf{s}}\left(\xi_{0}\right) \times \mathbf{s}^{\prime}\left(\xi_{0}\right)
$$

Перейдем теперь к вычислению вклада, вносимого появляющейся после разложения выражения (П.1) суммой:

$$
\frac{\Lambda}{\Lambda^{2}+1} \int d^{2} \mathbf{x}_{\perp}\left\{\left(\dot{\mathbf{s}}\left(\xi_{0}\right) \nabla_{\perp} \psi_{v}\left(\mathbf{x}_{\perp}\right)\right) \nabla_{\perp} \psi_{v}^{*}\left(\mathbf{x}_{\perp}\right)+\left(\dot{\mathbf{s}}\left(\xi_{0}\right) \nabla_{\perp} \psi_{v}^{*}\left(\mathbf{x}_{\perp}\right)\right) \nabla_{\perp} \psi_{v}\left(\mathbf{x}_{\perp}\right)\right\}
$$

Представим для удобства статическое решение $\psi_{v}\left(\mathbf{x}_{\perp}\right)$ в декартовых координатах $\eta_{1}, \eta_{2}$, лежащих в плоскости, перпендикулярной вихревой линии (и проходящей через точку $\left.\xi_{0}\right)$. Тогда

$$
\left(\dot{\mathbf{s}}\left(\xi_{0}\right) \nabla_{\perp} \psi_{v}\left(\mathbf{x}_{\perp}\right)\right) \nabla_{\perp} \psi_{v}^{*}\left(\mathbf{x}_{\perp}\right)=\left(\dot{\mathbf{s}}_{1} \frac{\partial \psi_{v}}{\partial \eta_{1}}+\dot{\mathbf{s}}_{2} \frac{\partial \psi_{v}}{\partial \eta_{2}}\right)\left(\frac{\partial \psi_{v}^{*}}{\partial \eta_{1}} \mathbf{e}_{1}+\frac{\partial \psi_{v}^{*}}{\partial \eta_{2}} \mathbf{e}_{2}\right) .
$$

Здесь $\dot{\mathbf{s}}_{1}, \dot{\mathbf{s}}_{2}-$ компоненты скорости вихревой линии в направлениях $\eta_{1}, \eta_{2}$, соответственно, а $\mathbf{e}_{1}, \mathbf{e}_{2}$ - единичные векторы в этих направлениях. Складывая выражение (П.6) с соответствующим комплексно-сопряженным, получим

$$
\begin{aligned}
& \mathbf{e}_{1}\left(2 \dot{\mathbf{s}}_{1} \frac{\partial \psi_{v}}{\partial \eta_{1}} \frac{\partial \psi_{v}^{*}}{\partial \eta_{1}}+\dot{\mathbf{s}}_{2} \frac{\partial \psi_{v}}{\partial \eta_{2}} \frac{\partial \psi_{v}^{*}}{\partial \eta_{1}} \dot{\mathbf{s}}_{2} \frac{\partial \psi_{v}}{\partial \eta_{1}} \frac{\partial \psi_{v}^{*}}{\partial \eta_{2}}\right)+ \\
& \quad+\mathbf{e}_{2}\left(2 \dot{\mathbf{s}}_{2} \frac{\partial \psi_{v}}{\partial \eta_{2}} \frac{\partial \psi_{v}^{*}}{\partial \eta_{2}}+\dot{\mathbf{s}}_{1} \frac{\partial \psi_{v}}{\partial \eta_{2}} \frac{\partial \psi_{v}^{*}}{\partial \eta_{1}}+\dot{\mathbf{s}}_{1} \frac{\partial \psi_{v}}{\partial \eta_{2}} \frac{\partial \psi_{v}^{*}}{\partial \eta_{1}}\right)
\end{aligned}
$$

Комбинация

$$
\frac{\partial \psi_{v}}{\partial \eta_{2}} \frac{\partial \psi_{v}^{*}}{\partial \eta_{1}}+\frac{\partial \psi_{v}}{\partial \eta_{1}} \frac{\partial \psi_{v}^{*}}{\partial \eta_{2}}
$$

строго обрашается в нуль для статического решения $\psi_{v}\left(\mathbf{x}_{\perp}\right)$. Это легко видеть из того, что комплексное сопряжение эквивалентно замене $\eta_{1} \rightarrow-\eta_{2}$. Оставшиеся слагаемые 
представляют собой произведение скорости линии на $\left|\nabla_{\perp} \psi_{v}\left(\mathbf{x}_{\perp}\right)\right|^{2}$. После интегрирования получаем известный результат:

$$
\int d^{2} x_{\perp}\left|\nabla_{\perp} \psi_{v}\left(\mathbf{x}_{\perp}\right)\right|^{2}=2 \pi \rho_{s} \ln \frac{a_{0} R}{r_{0}}=2 \pi \rho_{s} \sigma
$$

где $a_{0}$ - некоторая (слабо зависящая от $R$ ) функция порядка единицы. Это означает, что искомый вклад в уравнение (6) есть

$$
\frac{2 \pi \rho_{s} \sigma \Lambda}{\Lambda^{2}+1} \dot{\mathbf{s}}\left(\xi_{0}\right)
$$

Сумма выражений (П.4) и (П.9) есть интеграл в левой части уравнения (6), записанньй в терминах переменной $\mathbf{s}(\xi, t)$, которая описывает вихревую конфигурацию.

Благодарности. Автор благодарен Э. Б. Сонину и Г. Вильямсу за обсуждение ряда вопросов. Работа выполнена при финансовой поддержке INTAS (грант № 2001-0618) и РФФИ (грант № 03-02-16179).

\section{Список литературы}

[1] R. J. Donnelly. Quantized Vortices in Helium II. Cambridge: Camb. Univ. Press, 1991.

[2] W. H. Zurek. Phys. Rep. 1996. V. 276. № 4. P. 177; cond-mat/9607135.

[3] S. K. Nemirovskii, W. Fiszdon. Rev. Mod. Phys. 1995. V. 67. P. 37.

[4] Л. П. Питаевскиий. ЖЭТФ. 1958. Т. 35. С. 408.

[5] E.P. Gross. Nuovo Cimento. 1961. V. 20. P. 454.

[6] P. C. Hohenberg, B. I. Halperin. Rev. Mod. Phys. 1972. V. 49. P. 435.

[7] A. L. Fetter. Phys. Rev. 1966. V. 151. P. 100.

[8] L. M. Pismen. Vortices in Nonlinear Fields. Oxford: Clarendon Press, 1999.

[9] G. K. Batchelor. An Introduction to Fluid Mechanics. Cambridge: Camb. Univ. Press, 1967.

[10] J. Zinn-Justin. Quantum Field Theory and Critical Phenomena. Oxford: Clarendon Press, 1992.

Поступила в редакцию 11.VIII.2003 г. 